\title{
Thrombin Generation in Patients with Coronavirus Disease 2019
}

\author{
Marco Benati, PhD ${ }^{1}$ Gian Luca Salvagno, MD ${ }^{1} \quad$ Simone De Nitto, MD ${ }^{1}$ Matteo Gelati, $\mathrm{PhD}^{1}$ \\ Barbara Lavorgna, $\mathrm{MD}^{1}$ Cristiano Fava, $\mathrm{MD}^{2}$ Pietro Minuz, $\mathrm{MD}^{2, *}$ Giuseppe Lippi, MD ${ }^{1, *}$
}

1 Section of Clinical Biochemistry, University and Azienda Ospedaliera Universitaria Integrata of Verona, Verona, Italy

2 Division of General Medicine and Hypertension, University and Azienda Ospedaliera Universitaria Integrata of Verona, Verona, Italy

Semin Thromb Hemost 2021;47:447-450.

Several lines of evidence garnered so far attest that coronavirus disease 2019 (COVID-19) is associated with a remarkably high rate of thrombotic events. ${ }^{1}$ Di Minno et al recently published the results of a critical literature review and meta-analysis, including 20 studies and totaling 1988 COVID-19 patients, ${ }^{2}$ which revealed that the weighted mean prevalence of venous thromboembolism (VTE), deep vein thrombosis (DVT), and pulmonary embolism (PE) was as high as 31.3\% (95\% confidence interval [95\% CI], 24.3-39.2\%), 19.8\% (95\% CI: $10.5-34.0 \%$ ), and $18.9 \%$ (95\% CI: $14.4-24.3 \%$ ), respectively. The presenting characteristics and outcomes of 455 patients with COVID-19 who developed VTE (83\% PE and 17\% isolated DVT, respectively) during hospitalization have also been recently described by Fernández-Capitán et al. ${ }^{3}$ Interestingly, these patients had a median age of 65 years and most (i.e., over $70 \%)$ were male. The most frequent comorbidity was hypertension ( $42 \%$ ), followed by diabetes (20\%), chronic pulmonary disorders (10\%), coronary artery disease (6\%), and $4 \%$ were active smokers. The vast majority of these patients were immobilized (nearly $80 \%$ ), while $4 \%$ were also diagnosed as having active cancer. Positive personal history for previous episodes of VTE was only present in less than $4 \%$ of all these patients. Irrespective of the high likelihood of developing VTE, DVT, PE and even in situ pulmonary thrombosis, ${ }^{4}$ COVID-19 patients seem to carry also a considerable enhanced risk of arterial thrombosis, as recently emphasized by Boonyawat et al. ${ }^{5}$ These authors performed a comprehensive meta-analysis, including 36 studies, and reporting that the incidence of arterial thromboembolism in COVID-19 was as around 3\% in intensive care unit (ICU) settings. In another recent metaanalysis, published by Kunutsor and Laukkanen, ${ }^{6}$ the incidence of myocardial infarction, ischemic stroke, and sys-

These authors share senior authorship on this work.

published online January 22, 2021
Issue Theme Maintaining Hemostasis and Preventing Thrombosis in COVID-19 -Part II; Guest Editors: Emmanuel J. Favaloro, PhD, FFSc (RCPA) and Giuseppe Lippi, MD.

Address for correspondence Marco Benati, PhD, Section of Clinical Biochemistry, University of Verona, Hospital "Policlinico G.B. Rossi,", P.le LA Scuro 10, 37134 Verona, Italy (e-mail: marco.benati@univr.it).

temic arterial embolism was found to be comprised between $1.6 \%$ and $3.3 \%$.

Although it seems then rather clear that COVID-19 is a systemic pathology characterized by a strong propensity toward developing micro- and macrothrombotic episodes within the lungs and in many other organs and tissues, ${ }^{7,8}$ doubts remain as to whether this notable propensity may be attributable to immunothrombosis, platelet hyperactivity, enhanced blood coagulation, or to a variable combination of all these factors. ${ }^{9,10}$ Therefore, this study was aimed to assess thrombin generation in COVID-19 patients compared with a cohort of healthy controls.

Citrate blood was collected by direct venipuncture from 16 patients hospitalized for COVID-19( $63 \pm 15$ years; 9 females, 56\%; 8/16 needing oxygen therapy; mean hospital stay, $12 \pm 6$ days) and 19 ostensibly healthy controls ( $34 \pm 7$ years; 7 females; $37 \%$ ) recruited from the medical staff. No patients or controls were taking anticoagulant or antiplatelet therapies. Blood was drawn into evacuated blood tubes containing 3.2\% buffered sodium citrate (Vacutest Kima, Padova, Italy). The samples were immediately conveyed to the local laboratory, centrifuged at $1,500 \times \mathrm{g}$ for 15 minutes, and plasma was then separated and stored in aliquots at $-70^{\circ} \mathrm{C}$ until further laboratory assessment. Thrombin generation was assayed in all plasma aliquots with duplicate measures (final results were presented as mean of the duplicates) with STGThromboScreen, using the fully-automated ST Genesia analyzer (Diagnostica Stago, Asnières, France). The specific characteristics of this assay, performed either with or without thrombomodulin (TM), have been previously described elsewhere. ${ }^{11}$ The following parameters of thrombin generation were recorded in all participants: lag time (LT), peak height $(\mathrm{PH})$ of thrombin generation, time to reach the peak (TP) of thrombin generation, and endogenous thrombin potential

(c) 2021. Thieme. All rights reserved. Thieme Medical Publishers, Inc., 333 Seventh Avenue, 18th Floor, New York, NY 10001, USA
DOI https://doi.org/ 10.1055/s-0041-1722844. ISSN 0094-6176. 
(ETP). Results were shown as median and interquartile range. The significance of differences was assessed with MannWhitney $U$ test and Wilcoxon signed rank tests, when appropriate. Spearman's test was used for correlation analysis. The study was cleared by the Ethical Committee of Verona and Rovigo provinces (2577CESC clinical data registry and 2622CESC laboratory research). All subjects provided a written informed consent for participating in this study.

The results of this investigation are shown in - Table 1 and -Fig. 1. As compared with the healthy control group, the values of LT ( 2.68 vs. 1.87 minute; $p<0.001)$ and TP ( 4.60 vs. 4.08 minute; $p=0.031$ ) were found to be significantly enhanced in COVID-19 patients. Similar results were found with using the TM-supplemented assay (LT: 2.71 vs. 1.90 minutes; $p<0.001$; TP: 4.69 vs. 3.81 minutes; $p=0.001$ ). Unlike these two parameters, PH and ETP were found to not be significantly different between cases and controls with or without TM supplementation ( - Table 1 and $\boldsymbol{- F i g . ~} \mathbf{1}$ ). A high correlation was found between thrombin generation parameters assessed with or without TM in COVID-19 patients, as follows, LT: $r=0.97$ (95\% CI: 0.91-0.99; $p<0.001$ ); PH: $r=0.98$ (95\% CI: 0.94-0.99; $p<0.001$ ); TM: $r=0.96$ (95\% CI: 0.89-0.99; $p<0.0001$ ); ETP: $r=0.87$ (95\% CI: $0.65-0.95 ; p<0.001$ ). A marginally significant inverse correlation was found between the days of hospital stay and ETP without TM $(r=-0.50 ; 95 \% \mathrm{CI}$ : -0.80 to $-0.01 ; p=0.048)$, while a borderline significance was noted with ETP with TM $(r=-0.47 ; 95 \% \mathrm{CI}$ : -0.78 to 0.03 ; $p=0.066$ ). The association between length of hospital stay and other thrombin generation parameters did not achieve statistical significance. Finally, the ratio calculated between thrombin generation parameters measured with or without TM in all plasma samples did not significantly differ between the two cohorts of COVID-19 patients and healthy controls (data not shown).

The findings of normal or even decreased TG according to the assessed parameters in our patients hospitalized for severe COVID-19 illness could be considered almost unpredictable according to the commonplace perception of this condition as a prothrombotic disorder. To this end, however, quite similar findings have been published by White et al, ${ }^{12}$ who measured thrombin generation on Stago Genesia in 109 COVID-19 patients (75 with critical illness). In keeping with our findings, the values of LT and TP (with or without $\mathrm{TM}$ ) were also found to be higher in critical patients, while no major differences were seen in PH or ETP. In another study, Nougier et al assessed thrombin generation with calibrated automated thrombography in 78 COVID-19 patients (48 needing intensive care). ${ }^{13}$ Interestingly, prothrombin time was found to be lower in COVID-19 patients with critical illness compared with those with milder disease, while ETP values were overlapping. Conversely, impaired fibrinolysis measured with rotational thromboelastometry was commonplace in patients severe COVID-19 illness. Other studies have been published using indirect biomarkers of thrombin generation, but discussion of these findings may be inappropriate since they would not directly compare with ours or others, based on an automated thrombin generation assay.
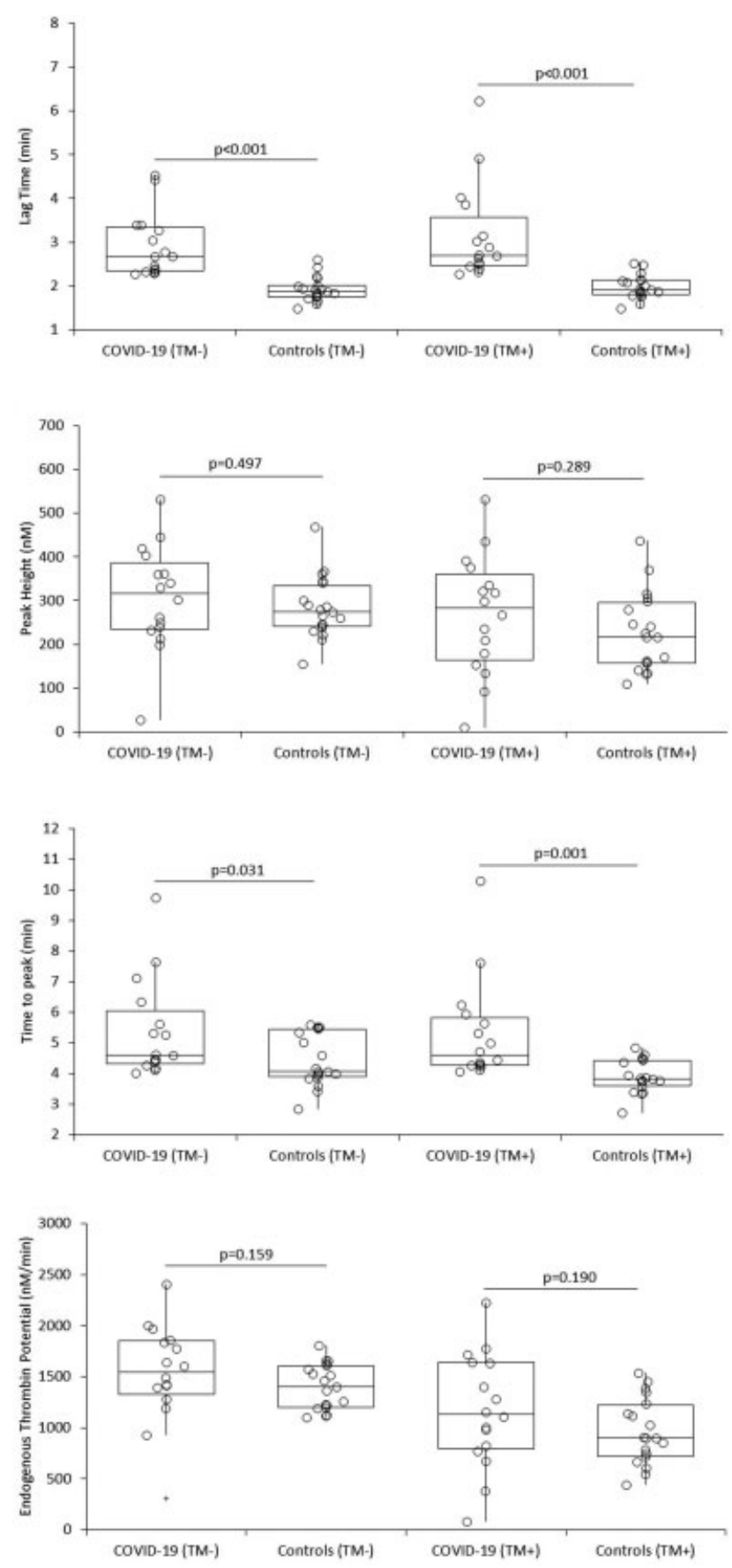

Fig. 1 Comparison of lag phase (LP), peak height of thrombin generation (PH), time to reach the peak of thrombin generation (TP), and endogenous thrombin potential (ETP) between patients with coronavirus disease 2019 (COVID-19) and ostensibly healthy controls recruited from the medical staff. Values are shown as median and interquartile range. $\mathrm{TM}+$, with thrombomodulin; $\mathrm{TM}-$, without thrombomodulin.

Taken together, these and previous findings are suggestive of some degrees of coagulation exhaustion in COVID-19, at least at a stage of disease needing oxygen therapy and/or intensive care, which would hence confirm the existence of initial local and/or systemic activation of blood coagulation, followed by significant exhaustion, as earlier noted in other studies, even using different markers. ${ }^{12-15}$ These findings are also in keeping with solid evidence of prolonged 
Table 1 Values (median and interquartile range) of thrombin generation parameters in patients with COVID-19 ( $n=16)$ and ostensibly healthy controls $(n=19)$

\begin{tabular}{|c|c|c|c|c|c|c|}
\hline \multirow[t]{2}{*}{ Parameters } & COVID-19 & Healthy controls & $p$-Value & COVID-19 & Healthy controls & $p$-Value \\
\hline & \multicolumn{3}{|c|}{ Without thrombomodulin } & \multicolumn{3}{|c|}{ With thrombomodulin } \\
\hline $\begin{array}{l}\text { Lag time } \\
\text { (min) }\end{array}$ & $\begin{array}{l}2.68 \\
(2.27-4.53)\end{array}$ & $\begin{array}{l}1.87 \\
(1.49-2.60)\end{array}$ & $\mathrm{p}<0.001$ & $\begin{array}{l}2.71 \\
(2.28-6.23)\end{array}$ & $\begin{array}{l}1.9 \\
(1.48-2.52)\end{array}$ & $p<0.001$ \\
\hline $\begin{array}{l}\text { Peak height } \\
(\mathrm{nM})\end{array}$ & $\begin{array}{l}316.85 \\
(28.61-530.9)\end{array}$ & $\begin{array}{l}265.4 \\
(156.3-468.3)\end{array}$ & $p=0.497$ & $\begin{array}{l}283.15 \\
(10.37-532.20)\end{array}$ & $\begin{array}{l}217.6 \\
(110.1-437.8)\end{array}$ & $p=0.289$ \\
\hline $\begin{array}{l}\text { Time to } \\
\text { peak (min) }\end{array}$ & $\begin{array}{l}4.60 \\
(4.02-9.74)\end{array}$ & $\begin{array}{l}4.08 \\
(2.84-5.59)\end{array}$ & $p=0.031$ & $\begin{array}{l}4.69 \\
(4.06-10.29)\end{array}$ & $\begin{array}{l}3.81 \\
(2.72-4.85)\end{array}$ & $p=0.001$ \\
\hline $\operatorname{ETP}(\mathrm{nM} / \mathrm{min})$ & $\begin{array}{l}1547 \\
(302.8-2404)\end{array}$ & $\begin{array}{l}1365 \\
(1103-1808)\end{array}$ & $p=0.159$ & $\begin{array}{l}1132.5 \\
(80.27-2226)\end{array}$ & $\begin{array}{l}896.6 \\
(442.1-1533)\end{array}$ & $p=0.190$ \\
\hline
\end{tabular}

Abbreviations: COVID-19, coronavirus disease 2019; ETP, endogenous thrombin potential.

Note: Bold values indicate statistical significance.

prothrombin times and decreased platelet counts in COVID19 patients, especially those progressing to severe/critical illness, as underpinned in most recent meta-analyses. ${ }^{16-18}$ It is also noteworthy that the almost unvaried evidence in thrombin generation values performed with or without TM would suggest that the protein $C$ system may be a minor player on COVID-19 coagulopathy, while activation of platelets and the factor XII-dependent pathway may alternatively appear as major drivers. ${ }^{14}$ Alternate observations of an enhanced rather than a smooth or even decreased thrombin generation may also be reflective of a timeline situation, since different findings would be expected across different phases of COVID-19, from asymptomatic illness to development of episodes of venous and/or arterial thrombosis. ${ }^{7}$ This aspect has been clearly emphasized by Hardy et al, ${ }^{19}$ who followed up 21 patients with COVID-19 for up to 30 days of ICU stay. The ETP values were found to be considerably increased upon ICU admission, but then progressively declined within, or even below, the normal range $\sim 10$ days afterward. This is in total agreement our findings that ETP was inversely associated with the length of hospital stay in our patients.

Notably, this investigation was not originally planned as cross-sectional study, as the healthy population was selected for obtaining local reference values for the thrombin generation assay. Although additional studies would hence be needed for addressing the potential contribution of age and other comorbidities on the thrombin generation tests used in this study, the significant age difference between cases and controls would probably not be sufficient to fully explain our findings, since no major impact of aging has been reported on ST Genesia ThromboScreen in the recent study of Calzavarini et al. ${ }^{20}$

Conflict of Interest

None declared.

\section{References}

1 Favaloro EJ, Lippi G. Maintaining hemostasis and preventing thrombosis in coronavirus disease 2019 (COVID-19)-part I. Semin Thromb Hemost 2020;46(07):757-762
2 Di Minno A, Ambrosino P, Calcaterra I, Di Minno MND. COVID-19 and venous thromboembolism: a meta-analysis of literature studies. Semin Thromb Hemost 2020;46(07):763-771

3 Fernández-Capitán C, Barba R, Díaz-Pedroche MDC, et al. Presenting characteristics, treatment patterns, and outcomes among patients with venous thromboembolism during hospitalization for COVID-19. Semin Thromb Hemost 2020 (e-Pub ahead of print). Doi: $10.1055 / \mathrm{s}-0040-1718402$

4 Thachil J, Srivastava A. SARS-2 coronavirus-associated hemostatic lung abnormality in COVID-19: is it pulmonary thrombosis or pulmonary embolism? Semin Thromb Hemost 2020;46(07): 777-780

5 Boonyawat K, Chantrathammachart P, Numthavej P, et al. Incidence of thromboembolism in patients with COVID-19: a systematic review and meta-analysis. Thromb J 2020;18(01):34

6 Kunutsor SK, Laukkanen JA. Incidence of venous and arterial thromboembolic complications in COVID-19: a systematic review and meta-analysis. Thromb Res 2020;196:27-30

7 Lippi G, Sanchis-Gomar F, Henry BM. COVID-19: unravelling the clinical progression of nature's virtually perfect biological weapon. Ann Transl Med 2020;8(11):693

8 Schulman S. Coronavirus disease 2019, prothrombotic factors, and venous thromboembolism. Semin Thromb Hemost 2020;46 (07):772-776

9 Vadasz Z, Brenner B, Toubi E. Immune-mediated coagulopathy in COVID-19 infection. Semin Thromb Hemost 2020;46(07): $838-840$

10 Parra-Izquierdo I, Aslan JE. Perspectives on platelet heterogeneity and host immune response in coronavirus disease 2019 (COVID19). Semin Thromb Hemost 2020;46(07):826-830

11 Salvagno GL, Favaloro EJ, Demonte D, et al. Influence of hypertriglyceridemia, hyperbilirubinemia and hemolysis on thrombin generation in human plasma. Clin Chem Lab Med 2019;57(11): 1784-1789

12 White D, MacDonald S, Edwards T, et al. Evaluation of COVID-19 coagulopathy; laboratory characterization using thrombin generation and nonconventional haemostasis assays. Int J Lab Hematol 2020 (e-Pub ahead of print). Doi: 10.1111/ijlh.13329

13 Nougier C, Benoit R, Simon M, et al. Hypofibrinolytic state and high thrombin generation may play a major role in SARS-COV2 associated thrombosis. J Thromb Haemost 2020;18(09): 2215-2219

14 Taus F, Salvagno G, Canè $S$, et al. Platelets promote thromboinflammation in SARS-CoV-2 pneumonia. Arterioscler Thromb Vasc Biol 2020;40(12):2975-2989

15 Wool GD, Miller JL. The impact of COVID-19 disease on platelets and coagulation. Pathobiology 2021;88(01):15-27 
16 Zhang X, Yang X, Jiao H, Liu X. Coagulopathy in patients with COVID-19: a systematic review and meta-analysis. Aging (Albany NY) 2020;12(24):24535-24551

17 Zhang A, Leng Y, Zhang Y, et al. Meta-analysis of coagulation parameters associated with disease severity and poor prognosis of COVID-19. Int J Infect Dis 2020;100:441-448

18 Di Minno MND, Calcaterra I, Lupoli R, et al. Hemostatic changes in patients with COVID-19: a meta-analysis with meta-regressions. J Clin Med 2020;9(07):2244
19 Hardy M, Michaux I, Lessire S, et al. Prothrombotic disturbances of hemostasis of patients with severe COVID-19: a prospective longitudinal observational study. Thromb Res 2020; 197:20-23

20 Calzavarini S, Brodard J, Quarroz C, et al. Thrombin generation measurement using the ST Genesia Thrombin Generation System in a cohort of healthy adults: normal values and variability. Res Pract Thromb Haemost 2019;3(04):758-768 